


\section{DOCENCIA, UN CAMPO DE ACCIÓN LABORAL PARA EL DESARROLLO DE LA PROFESIÓN DE FISIOTERAPIA} ○

\section{TEACHING, A FIELD OF LABOR ACTION FOR THE DEVELOPMENT OF THE PROFESSION OF PHYSIOTHERAPY}

EMERSON JULIÁN RINCON CASTILLO

\section{RESUMEN}

La profesión de fisioterapia en el mundo en las últimas décadas ha experimentado una transformación académica, lo cual ha conllevado al mejoramiento del perfil profesional y ocupacional, abriéndose camino en diferentes campos de acción. En la actualidad la fisioterapia es una profesión liberal, en la cual predomina el ejercicio intelectual, el conocimiento y la técnica, involucrando sus acciones a nivel clínico, administrativo e investigativo teniéndose en cuenta el contexto del país y las tendencias a nivel internacional. Objetivo: Reflexionar acerca del perfil profesional del fisioterapeuta en el campo de acción de la docencia. Método: El enfoque de este artículo es cualitativo, se usó como diseño metodológico de recolección de datos instrumento tipo encuesta, las cuales fueron dirigidas a fisioterapeutas docentes del programa de fisioterapia de la Corporación Universitaria Iberoamericana. Discusión: Aunque la profesión de fisioterapia hace parte de las ciencias de la salud y a pesar de que su formación en esencia es a nivel clínico-asistencial, el campo de la docencia se ha convertido en una opción importante para el desarrollo del fisioterapeuta. Conclusiones: Se debe considerar la posibilidad de fortalecer la malla curricular en el pregrado de fisioterapia en el área de la pedagogía y la docencia.

PALABRAS CLAVE: Fisioterapia - Competencia - Profesional Enseñanza - Educación - Laboral.
ABSTRACT

The profession of physiotherapy in the world in recent decades has undergone an academic transformation, which has led to the improvement of the professional and occupational profile, making its way into different fields of action. At present, physiotherapy is a liberal profession, in which intellectual exercise, knowledge and technique predominate, involving its actions at a clinical, administrative, and research level, taking into account the country's context and international trends. Objective: To reflect on the professional profile of the physiotherapist in the field of teaching. Method: The approach of this article is qualitative, the survey type instrument was used as methodological design of data collection, which were directed to physical therapists of the physiotherapy program of the Iberoamerican University Corporation. Discussion: Although the profession of physiotherapy is part of the health sciences and despite the fact that its training is strong at the clinical-healthcare level, the teaching field has become an important option for the development of the physiotherapist. Conclusions: The possibility of strengthening the curricular mesh in the undergraduate of physiotherapy in the area of pedagogy and teaching should be considered.

KEYWORDS: Therapy - Competence - Teaching - Education Working-Physical. 


\section{L}

a profesión de fisioterapia en el mundo en las últimas décadas ha experimentado una transformación académica, lo cual ha conllevado al mejoramiento del perfil profesional y ocupacional, abriéndose camino en diferentes campos de la acción. En la actualidad, la fisioterapia es una profesión liberal, en la cual predomina el ejercicio intelectual, el conocimiento y la técnica, involucrando sus acciones a nivel clínico asistencial, administrativo e investigativo, teniendo en cuenta el contexto del país y las tendencias a nivel internacional.

En la actualidad, el campo de la docencia se ha convertido en un campo de acción laboral de gran importancia para el fisioterapeuta, complementando las competencias laborales anteriormente mencionadas.

En Colombia, la Ley 528 (1999) del 14 de septiembre de 1999, reglamentó el ejercicio de la profesión de fisioterapia, y dictó normas en materia de ética profesional y otras disposiciones. En su Artículo 2, Literal g, establece lo siguiente:

La actividad pedagógica del fisioterapeuta es una noble práctica que debe ser desarrollada transmitiendo conocimientos y experiencias al paso que ejerce la profesión, 0 bien en función de la cátedra en instituciones universitarias u otras cuyo funcionamiento esté legalmente autorizado. En uno y otro caso, es deber suyo observar los fundamentos pedagógicos y un método de enseñanza que se ajuste a la ética profesional.

Es muy pertinente que la ley le otorgue al fisioterapeuta la posibilidad de desempeñarse en el campo de la

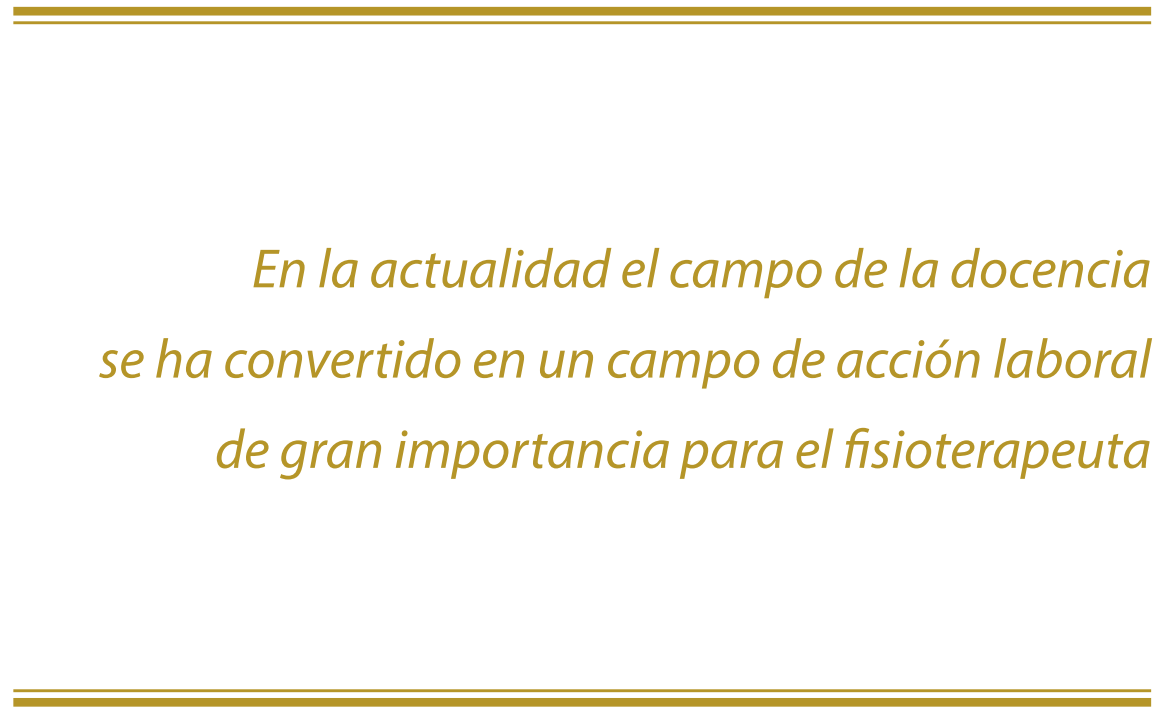

docencia. Sin embargo, esta actuación debe realizarse con responsabilidad, para lo cual es muy significativa la adquisición de capacidades y competencias en este campo de acción.

Es así como Quiroz, Durán, y Quiñones, (2012) en su artículo consideran que el fisioterapeuta debe mantener sus capacidades actualizadas; en Colombia el perfil profesional del fisioterapeuta se orienta en general hacia lo clínico, lo administrativo y lo investigativo. En referencia a la docencia los autores refieren que:

El enfoque de competencias profesionales en el ámbito educativo responde a una creciente demanda de la sociedad, en el sentido de conocer las capacidades que se desarrollan a través de los diferentes procesos de formación (p.157).

Lo anterior demuestra que la docencia es uno de los campos de acción en el cual el fisioterapeuta se ha empoderado y con el pasar de los años y su práctica profesional debe ser cada vez más especializada.

El perfil académico y ocupacional del fisioterapeuta egresado de la Univer- sidad Industrial de Santander (UIS), descrito mediante el uso de una encuesta a egresados, indicó que 51 participantes manifestaron haber adelantado estudios en Educación Continuada como Diplomado especialmente en las áreas de Rehabilitación y Neurodesarrollo, Gerencia y Administración de Servicios de Salud, Plan de Atención Básica, Educación y Docencia, Salud Ocupacional, Terapia Total y Epidemiología Clínica; un grupo de 39 fisioterapeutas acreditaron un nivel de especialización en las áreas de Docencia Universitaria, Salud Ocupacional, Pedagogía, Auditoría en Salud, Cuidado Crítico, Administración de Servicios de Salud, Técnica Deportiva y Gestión Pública, y 6 egresados acreditaron nivel de maestría en las áreas de Fisiología, Gestión y Dirección de Instituciones Educativas, Educación y Pedagogía. Esto permite establecer que la educación y la docencia son áreas de interés para el mejoramiento y profundización de las competencias del fisioterapeuta en Colombia.

El perfil profesional del fisioterapeuta en Colombia varía según el contexto social, cultural y económico de la región donde se desarrolle el profe- 
sional. Los campos de acción para el fisioterapeuta están en el sector de la promoción de la salud y prevención de la enfermedad, asistencia terapéutica, habilitación y rehabilitación, en el sector de la educación, áreas de desarrollo administrativo, de gestión e investigación.

En el artículo publicado por Casas y Ramírez (2015) establecen que las competencias específicas del fisioterapeuta se sitúan en la clínica, en la actividad física y el deporte, la salud y el trabajo, la educación y la investigación especialmente, así como el desarrollo de competencias en administración y gestión de servicios de salud.

El estudio de Casas y Ramírez (2015) establece que el fisioterapeuta en Colombia debe obtener competencias no únicamente en el área disciplinar clínico-asistencial, sino que es necesaria la adquisición de nuevos conocimientos que alimenten las competencias profesionales ajenas a lo clínico asistencial, como lo es el área de la educación.

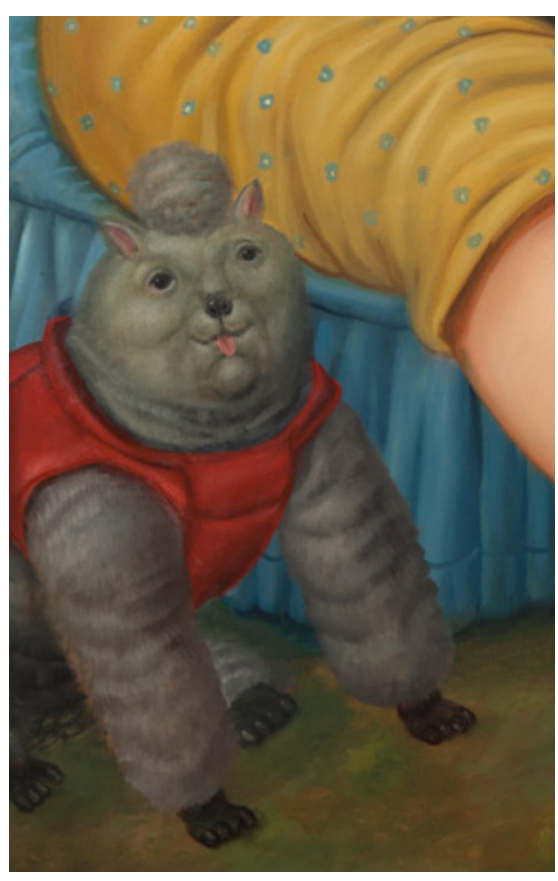

Rodríguez y Sarmiento (2012) identificaron 93 programas de fisioterapia ofertados por universidades localizadas en las regiones nororiental, occidental y sur de Europa y las categorías de análisis determinadas y emergentes para los perfiles de formación, datos pertinentes para este estudio. $A$ partir de la búsqueda realizada por los autores de las 93 universidades que ofertan el programa de fisioterapia en los países de Europa, se logró identificar los perfiles de formación, siendo el perfil clínico asistencial el de mayor importancia, seguido de los perfiles en rehabilitación deportiva, salud ocupacional, docencia e investigación. Este estudio demuestra que no solo en Colombia la docencia es un campo de acción laboral para el fisioterapeuta, ésta es una tendencia global.

De la Torre (2012) analizó el perfil de egreso de los estudiantes del programa de Terapia Física y Rehabilitación de la universidad católica Santiago de Guayaquil en Ecuador, mediante el uso de encuestas y entrevistas; propuso un rediseño curricular que permitió in-

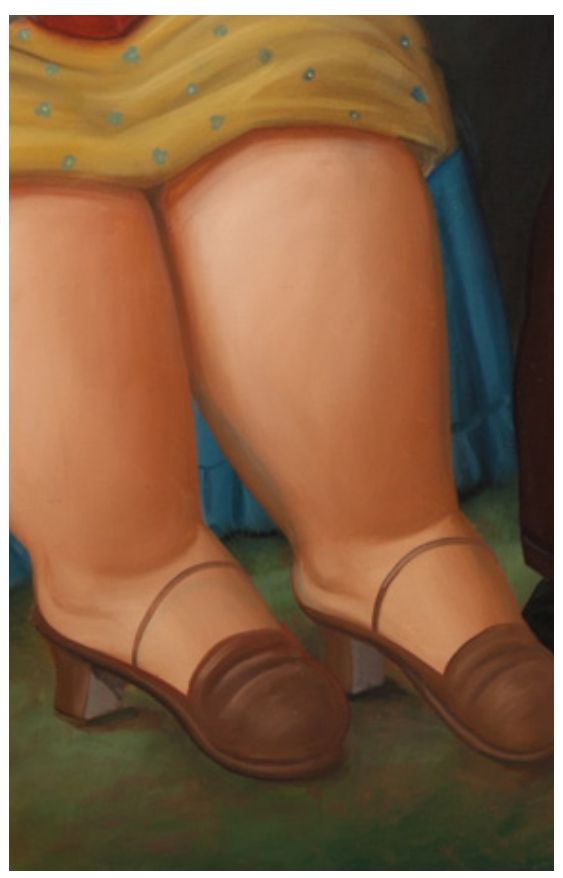

cursionar al fisioterapeuta en campos de acción poco explorados con el fin de brindarle mayores oportunidades de desempeño laboral. Propuso como competencia básica para la carrera de fisioterapia el cumplimiento de funciones a nivel asistencial, docencia y de investigación. El perfil del egresado de fisioterapia debe permitirle ejercer liderazgo en la terapia física en el sector privado y público de la salud, y su desempeño profesional es complementado en las áreas de atención al paciente, administrativa gerencial, educativa e investigativa.

La distribución del porcentaje de tiempo laboral dedicado a los campos de acción de los fisioterapeutas egresados de la población estudiada en la Universidad CES Medellín se desempeñan en distintas áreas: asistencial, educación, investigación y administración. Siendo el egresado CES un profesional joven con un gran interés en el escenario laboral educativo. Muñoz (2013), argumenta que los datos obtenidos tienen gran relación y convergencia con los datos logrados por el estudio nacional realizado por la Asociación Colombiana de Fisioterapeutas (ASCOFI), la Asociación Colombiana de Facultades de Fisioterapia (ASCOFAFI), Colegio Colombiano de Fisioterapeutas (COLFI) y la Asociación Colombiana de Estudiantes de Fisioterapia (ACEFIT).

La Gráfica 1 muestra datos suministrados por 29 programas de fisioterapia del país sobre el número de egresados entre los años 1980 y 2013, refiere que en Colombia se cuenta con cerca de 25,708 profesionales en ejercicio activo. Dentro de los escenarios del desempeño laboral el $24.1 \%$ lo hace en instituciones de educación 
universitaria, siendo la segunda área de desempeño laboral después de la atención domiciliaria clínica con un $33.2 \%$. Ministerio de salud y protección social; Asociación Colombiana de Colombiano de Fisioterapia; Colegio Fisioterapeutas, (2015).

Debe existir una coherencia entre la formación y el perfil ocupacional, y establecer la necesidad de revisar la estructura curricular y el plan de estudios con el fin de mejorar el desarrollo de competencias relacionadas con la educación, la administración y otras áreas de menor desempeño laboral. Se ha encontrado que el $64.3 \%$ se desempeña en el área asistencial, un $19 \%$ se desempeña en 20 más áreas, y un $14.4 \%$ en promoción y prevención, docencia y áreas de la administración. Chamorro, Paz y Vernaza (2009).

La literatura revisada permite estatuir que efectivamente el campo de la docencia es fuente importante de desarrollo laboral para el fisioterapeuta a nivel nacional y mundial, y ello permite dilucidar que de una u otra manera los programas de pregrado de fisioterapia deben de incluir dentro de su formación, cátedras que promuevan el desarrollo del conocimiento y de competencias en el área de la docencia, a fin de certificar en el perfil profesional del fisioterapeuta la competencia para desempeñarse en el campo de la educación y la docencia.

\section{MÉTODO}

El enfoque de este artículo es cualitativo, puesto que los estudios cualitativos se optan por sus propiedades explicativas y su poder exploratorio. Estos ayudan a esclarecer los resultados obtenidos en investigaciones cuantitativas o generar teorías en

Grafica 1. Escenarios donde el fisioterapeuta realiza su actividad laboral.

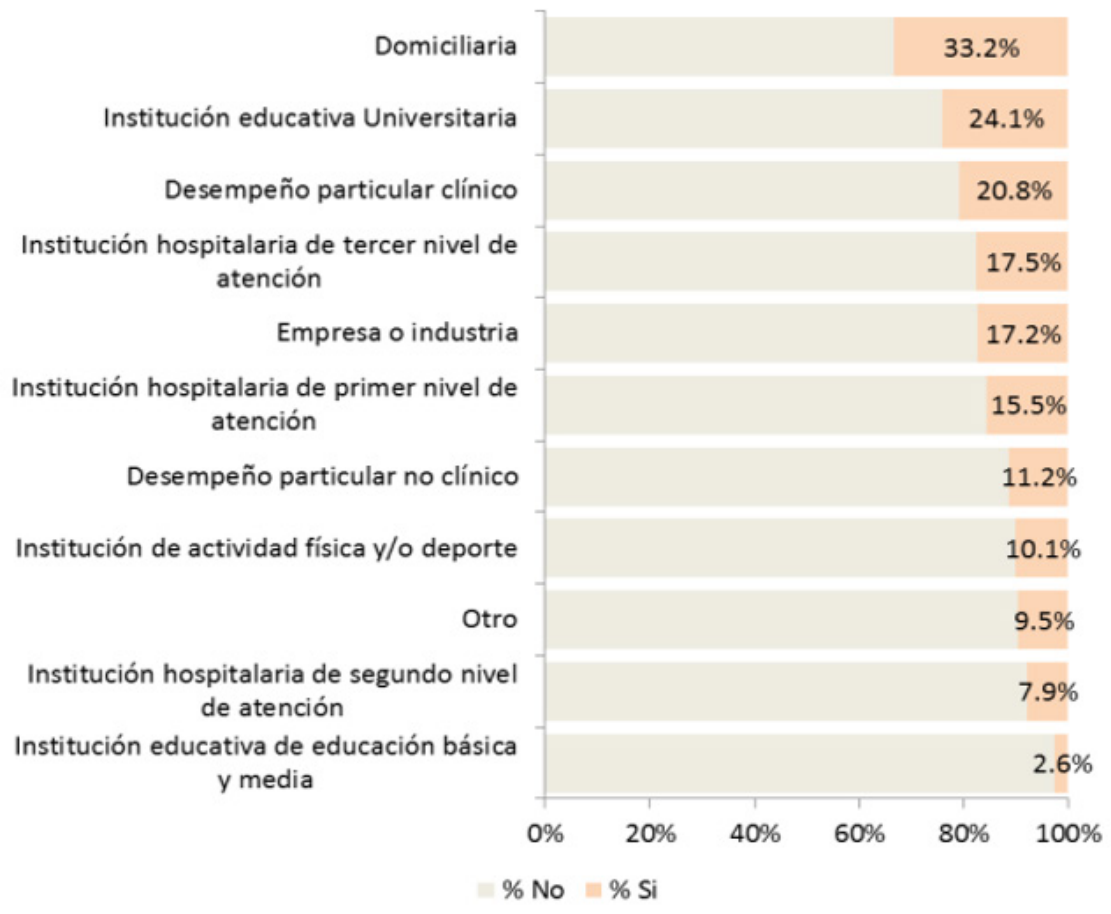

Fuente: Ministerio de salud y protección social; Asociación Colombiana de Fisioterapia; Colegio Colombiano de Fisioterapeutas, (2015).

campos poco explorados. Los métodos cualitativos son paradigmas, aplicados comúnmente en las ciencias sociales, donde los fenómenos no se pueden comprender en toda su amplitud desde información cuantitativa (Binda y Benavent, 2013).

Este trabajo es cualitativo en tanto que busca reflexionar acerca de la necesidad de fortalecer el perfil profesional del fisioterapeuta en el campo de acción de la docencia. Para este estudio de características cualitativas, se usó como diseño metodológico de recolección de datos el instrumento tipo encuesta, las cuales fueron dirigidas a fisioterapeutas docentes del programa de fisioterapia de la Corporación Universitaria Iberoamericana, con el objetivo de reflexionar acerca del perfil profesional del fisioterapeuta en el campo de acción de la docencia.
La población objeto de este estudio son los 65 docentes fisioterapeutas que hacen parte del programa de fisioterapia de la facultad de salud de la Corporación Universitaria Iberoamericana de la ciudad de Bogotá. Una vez definida la población, se estableció como muestra 20 fisioterapeutas docentes que hagan parte del programa de fisioterapia de la facultad de salud de la Corporación Universitaria Iberoamericana, los cuales se escogieron aleatoriamente.

\section{RESULTADOS}

Participaron 20 docentes del programa de fisioterapia de la Corporación Universitaria Iberoamericana, los cuales diligenciaron el instrumento tipo encuesta, arrojando los siguientes datos: La encuesta fue respondida por 14 docentes de género femenino y 6 docentes de género masculino. 


\section{Gráfica 2. Género de los Participantes}

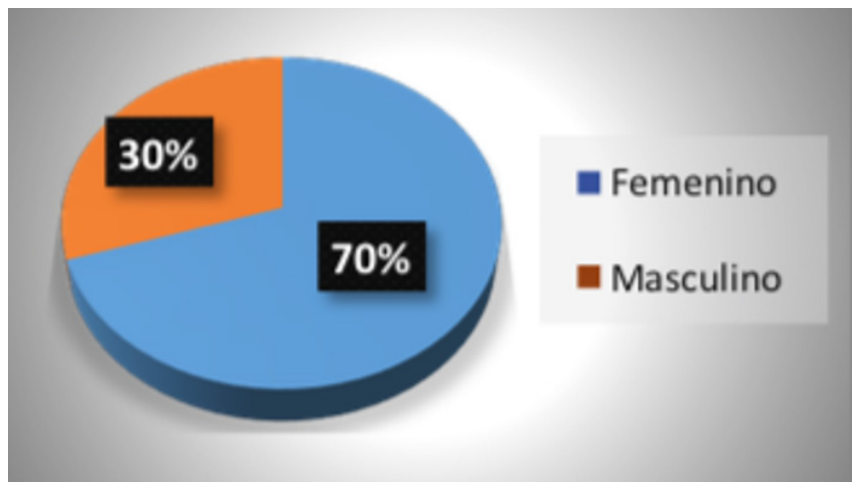

Tiempo de Experiencia. Ante la pregunta ¿cuál es el tiempo de experiencia en la docencia?, un $30 \%$ equivalente a 6 docentes tienen una experiencia en docencia entre 1 a 3 años, 5 docentes correspondiente a un 25\% tienen una experiencia mayor a 10 años, y en igual medida 3 docentes tienen experiencia menor a un año, 3 docentes tienen experiencia entre 3 a 5 años, y 3 docentes entre 5 y 10 años de experiencia, cada uno de ellos con un porcentaje del $15 \%$. Es importante reconocer una experiencia significativa en la docencia en 9 docentes mayor a 5 años.

\section{Gráfica 3. Tiempo de experiencia en la docencia}

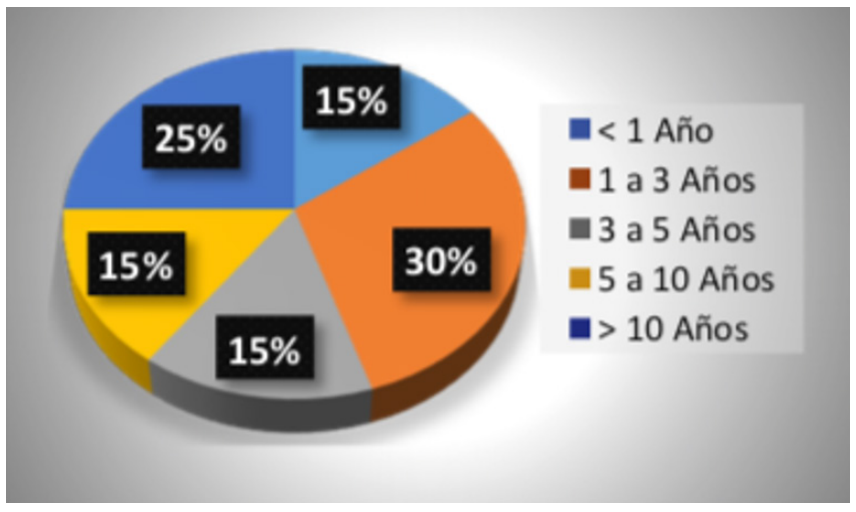

Deseo de desempeñarse como docente desde el pregrado. ante la pregunta ¿Desde el pregrado era su deseo desempeñarse como docente?, un número de 12 docentes correspondiente a un $60 \%$ respondieron negativamente, y 8 docentes lo que corresponde a un $40 \%$ respondieron en forma positiva.

\section{Gráfica 4. Deseo de desempeñarse como docente desde el pregrado}

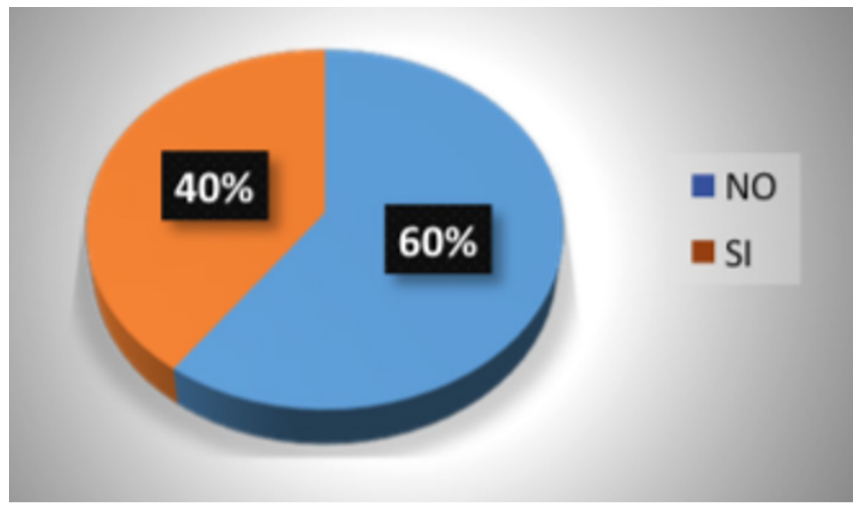

Docencia como primera opción laboral. Al preguntarse si la docencia fue su primera opción para laborar una vez egresaron como fisioterapeuta, se logró establecer que los 20 docentes, el $100 \%$ no contemplaron la docencia como primera opción laboral. Se logró determinar que la opción laboral mayoritaria fue el área clínico-asistencial.

\section{Gráfica 5. Docencia como primera opción laboral}

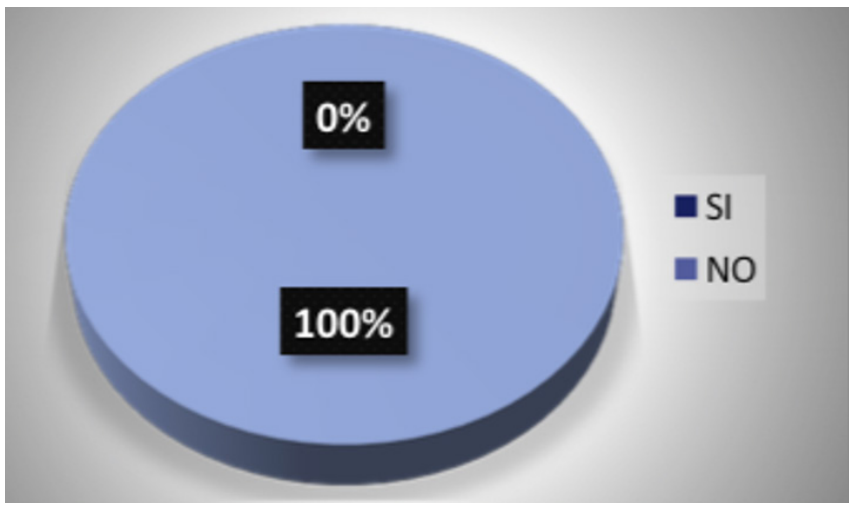


Formación en pedagogía o docencia en el pregrado. Al preguntarse sobre si habían recibido algún tipo de formación académica en pedagogía 0 en docencia durante la formación pre gradual en fisioterapia. 17 docentes un $85 \%$ respondieron que no habían recibido ningún tipo de formación pedagógica o en docencia durante el pregrado, sólo 3 docentes un $15 \%$ respondieron que sí habían recibido algún tipo de formación en pedagogía o en docencia.

\section{Gráfica 6. Recibieron formación en pedagogía o docencia en el pregrado}

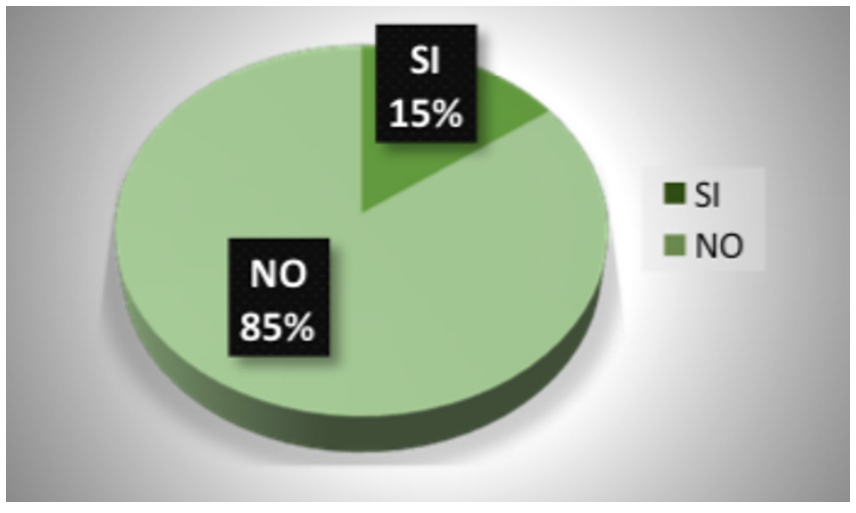

Consideran importante la formación en docencia desde el pregrado. Se preguntó si consideran importante la formación de competencia en pedagogía y/o docencia desde el pregrado. Los resultados mostraron que 16 docentes un $80 \%$ consideran importante la formación pregradual en fisioterapia y se contemple la formación en competencias para la docencia.

\section{Gráfica 7. Consideran importante la formación} en docencia desde el pregrado.

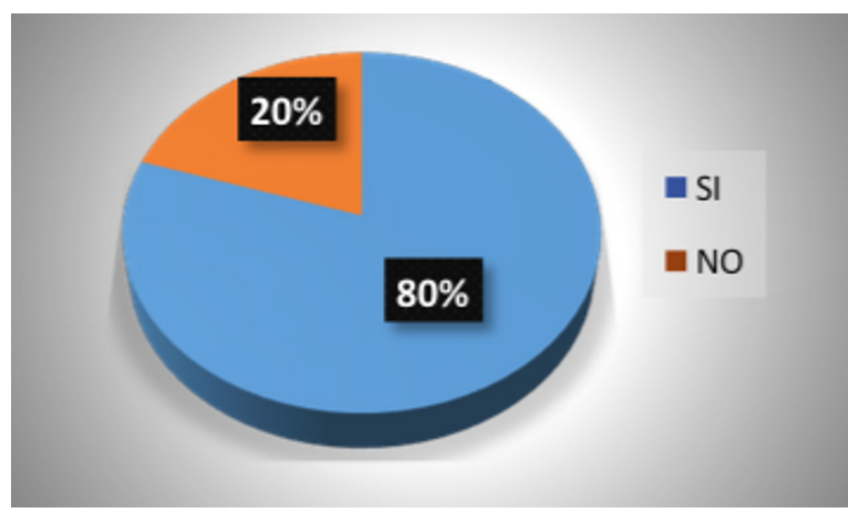

Docentes que cuentan con formación en Docencia. Se indagó a los docentes participantes sobre sí contaban con formación en docencia y qué tipo de formación habían cursado. De los 20 docentes participantes 13 docentes un $65 \%$ manifestaron no contar con formación en docencia, pero sí cuentan con formación post gradual en áreas afines a la disciplina de la fisioterapia 0 áreas afines a la salud. 7 docentes, un 35\%, respondieron que sí tenían formación en docencia. Así mismo, de estos 7 docentes, 6 han realizado diplomado en competencias docentes y uno respondió ser especialista en educación.

\section{Grafica 8. Docentes que cuentan con formación en Docencia}

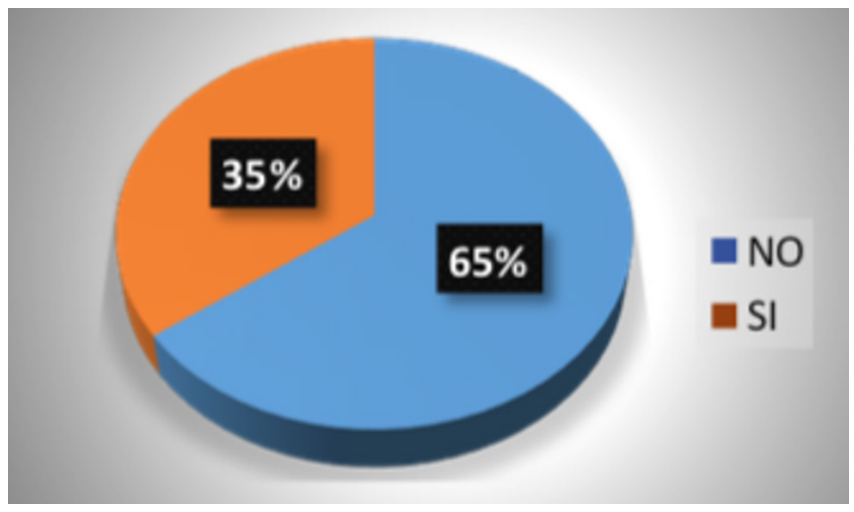

Gráfica 9. Tipo de Formación en Docencia

Tipo de Formación

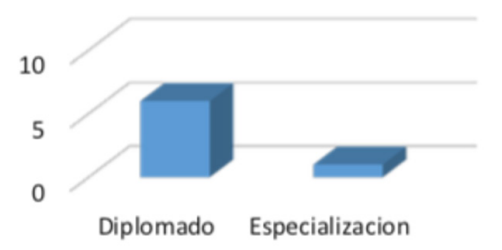


Satisfacción en su desempeño laboral como docente. Se preguntó a los docentes participantes en este estudio, si sienten que su labor como docentes es satisfactoria, el total de los docentes sienten gran satisfacción en su labor como docentes.

\section{Gráfica 10. Siente satisfacción en su desempeño laboral como docente}

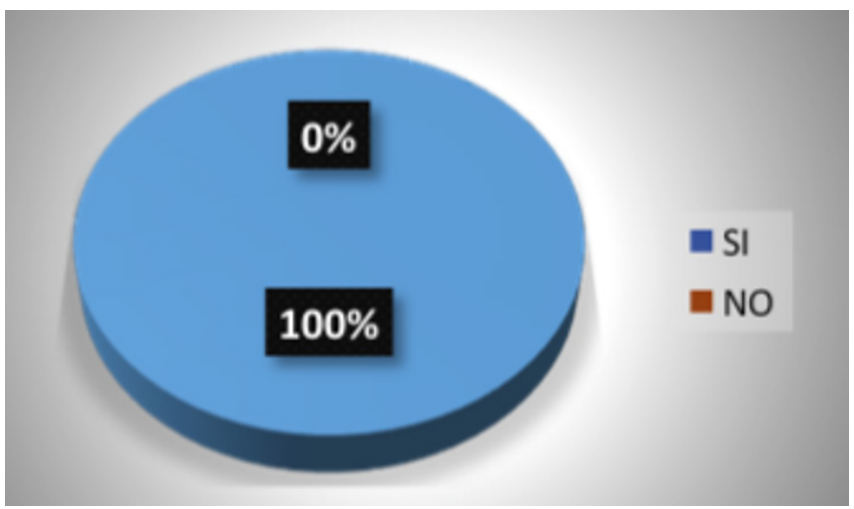

Incluir en la malla curricular la Formación en Competencias Docentes desde el Pregrado. Por último, se preguntó sobre si consideraban importante incluir en la malla curricular de fisioterapia asignaturas, cátedras o electivas que tengan como propósito la formación de competencias en docencia.Para la cual se obtuvo como resultado que 18 docentes un $90 \%$ están de acuerdo que se incluya en la malla curricular de los programas de fisioterapia asignaturas, cátedras o electivas dirigidas a la formación de competencias en pedagogía y/o docencia; 2 docentes representados con un $10 \%$ respondieron que no era importante esta inclusión en la malla curricular.

Gráfica 11. Consideran incluir en la malla curricular la Formación en Competencias Docentes desde el Pregrado
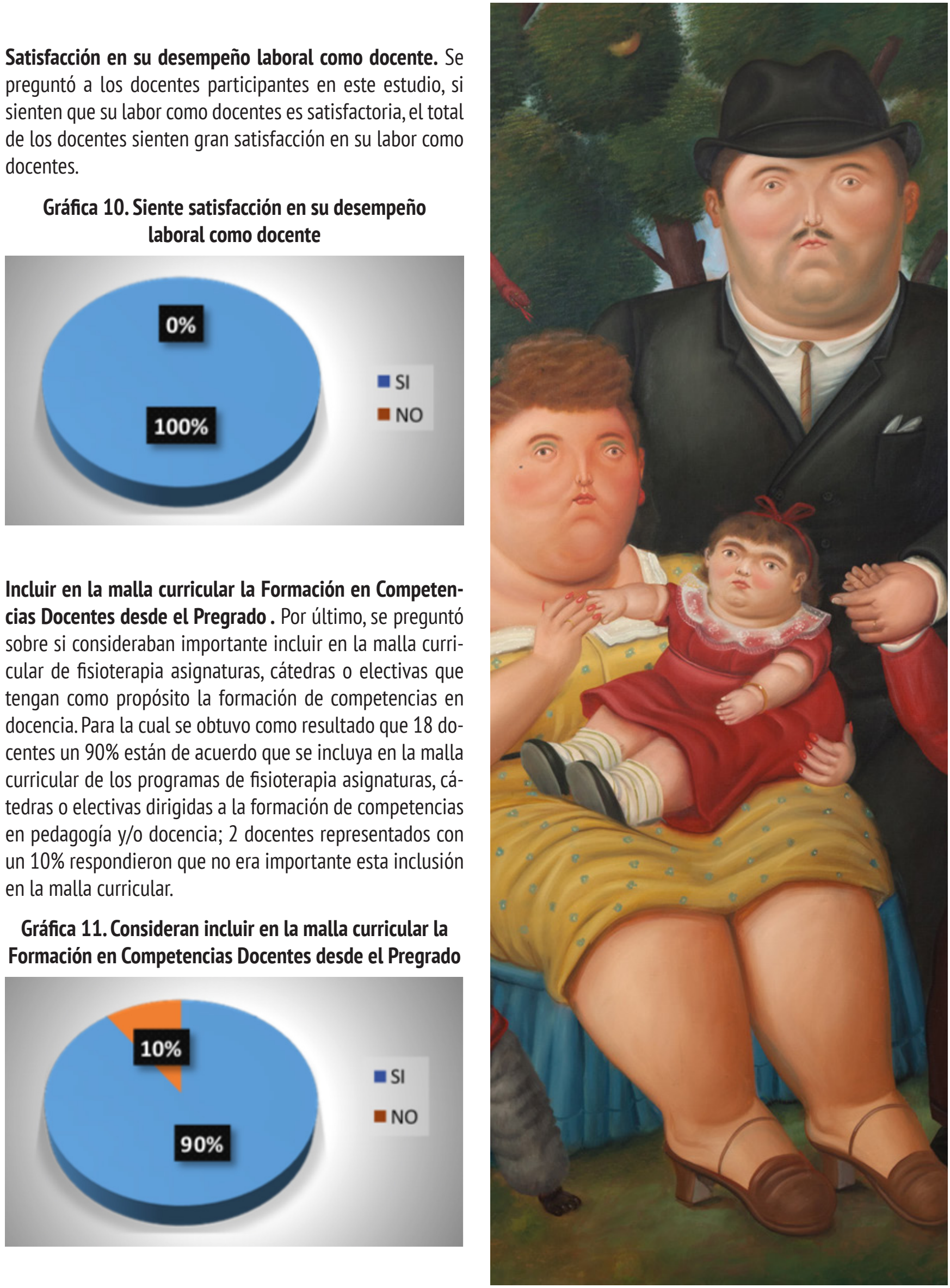
Tabla 1. Percepción de la docencia como campo de acción laboral para el fisioterapeuta

\section{Pregunta}

1. ¿Porque Eligió ser Docente?

Respuestas

Oportunidad de crecimiento

profesional. Conocimiento (Generar,

Aumentar, actualizado).

Reconocimiento. Orientación Familiar.

2. Si no deseaba ser docente en el pre grado y la docencia no fue su primera opción laboral, ¿Qué lo motivo a ejercer la docencia?

3. ¿Por qué considera que los fisioterapeutas deben tener formación en competencias docentes desde el pre grado?
Aprendizaje diario, es una bonita oportunidad de crecimiento profesional. Cambiar de ambiente laboral. Trasmitir la experiencias propias, Cambio de rol, Aportar experiencia a la profesión

Fortalecer su desempeño profesional, convenios relación servicio. Mejorar la didáctica, Favorecer el aprendizaje, porque siempre estamos enseñando algo a alguien, Integralidad de la profesión.

Fortalecimiento del rol y del desempeño profesional. Oportunidad de otro campo de acción laboral, Para que la docencia sea un campo de opción profesional desde el pregrado,

Contribuye a mejorar la calidad académica. Más herramientas para el desempeño como docente.

Agradecimiento. Cumplo como ser humano, Crecimiento personal, de los estudiantes y de la profesión.

5. ¿Por qué siente satisfacción en su labor como docente?

Contribuir a la profesión. Permite trasmitir mi experiencia profesional, Por que hago lo que me gusta que es enseñar.

Fuente: Elaboración Propia (2019)
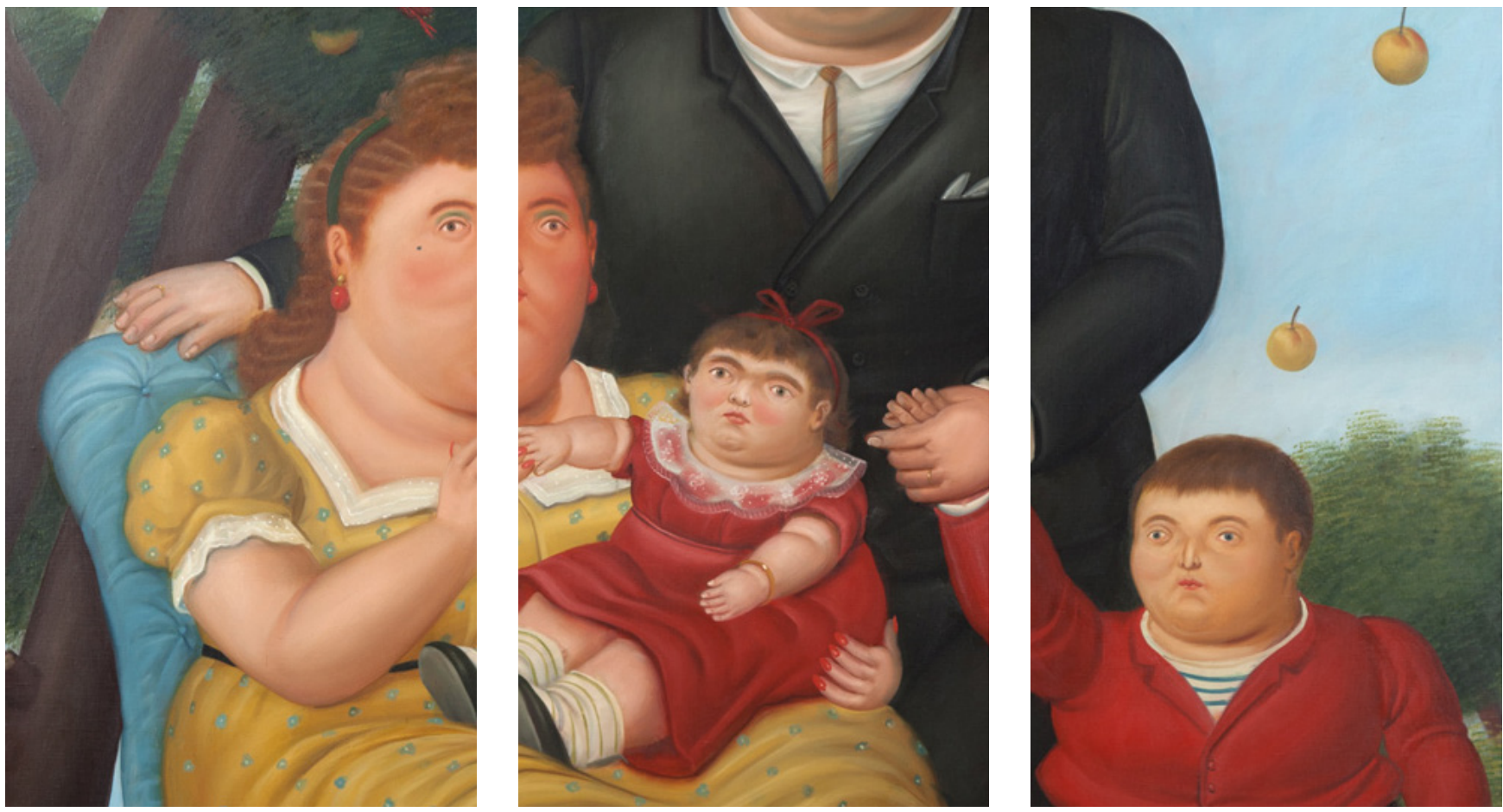

Presencia Universitaria 
Como se puede observar en la tabla 1 , los profesionales de fisioterapia perciben la docencia como una oportunidad de crecimiento profesional, al igual que una forma de perfeccionamiento académico, que conlleva a lograr un reconocimiento a nivel profesional y a una satisfacción personal. Así mismo, el cambio de rol profesional, la necesidad de generar conocimiento y hacer de sus experiencias una forma válida de enseñanza, son motivaciones para que el fisioterapeuta tome la decisión de ejercer la docencia.

Es importante reconocer que los docentes participes de este estudio consideran que es importante la formación de competencias en docencia desde el pregrado de fisioterapia, ya que ello contribuye a fortalecer el perfil profesional y la práctica profesional, favorece el proceso de enseñanza aprendizaje y ello contribuye a la calidad y el desarrollo de la profesión de la fisioterapia.

Aunado a la anterior, los docentes participantes en este estudio opinan que es pertinente incluir en la malla curricular de pregrado de fisioterapia asignaturas, cátedras 0 electiva que tengan como propósito la formación de competencia en pedagogía y/o docencia, ya que esta inclusión fortalece el perfil profesional y ocupacional en el fisioterapeuta, dando oportunidad de explorar e incursionar en otro campo de acción como lo es la docencia.

Por último, los docentes participes en este estudio sienten una gran satisfacción en su labor docente, perciben que están contribuyendo con la educación del país, que participan activamente en el crecimiento personal y profesional de sus estudiantes y esto los llena de sentimientos de gratitud. Conciben su práctica docente como una manera de dejar un legado a las nuevas generaciones.

\section{DISCUSIÓN}

Aunque la profesión de fisioterapia hace parte de las ciencias de la salud y a pesar de que su formación es fuerte a nivel clínico-asistencial, el campo de la docencia se ha convertido en una opción importante para el desarrollo laboral del fisioterapeuta, por lo que se requiere de la adquisición de competencias desde el pregrado. $\mathrm{A}$ pesar de que la docencia no se considera como una opción laboral en el pregrado, trae consigo grandes retos para poder enfrentarla con calidad y competencia. Por tanto, es importante dar la discusión académica acerca de complementar la formación pregradual en competencias para la docencia, su pertinencia y sus alcances.

\section{CONCLUSIONES}

Los datos obtenidos de los estudios revisados, demuestran que el campo de acción de la docencia es de gran importancia a nivel laboral para el fisioterapeuta. La docencia es un campo de acción de gran impacto laboral tanto a nivel nacional como mundial. La docencia se encuentra entre los 3 campos de acción laboral más relevantes para el ejercicio profesional del fisioterapeuta. Es evidente la necesidad de fortalecer el perfil profesional del fisioterapeuta en el campo de la docencia.

La docencia trae consigo gran reconocimiento profesional, y satisfacción personal para el fisioterapeuta docente. Mejorar las competencias en docencia en el fisioterapeuta contribuirá a la calidad académica de la profesión. Es importante considerar incluir en las mallas curriculares de los programas de fisioterapia de pregrado cátedras, asignaturas 0 electivas que tengan como propósito la formación de competencias en docencia.

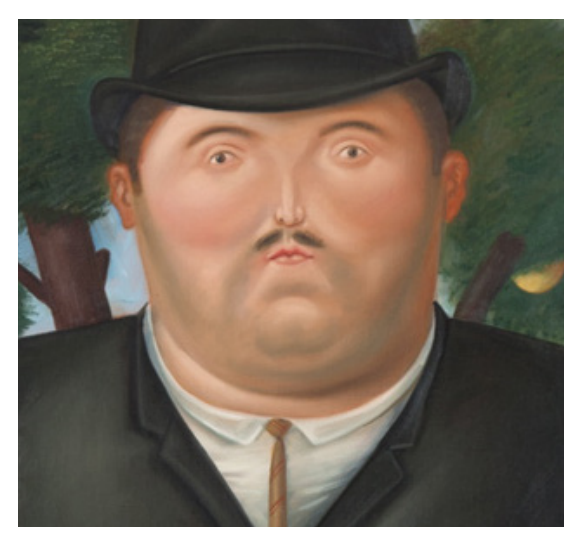

Los datos obtenidos de los estudios revisados, demuestran que el campo de acción de la docencia es de gran importancia a nivel laboral para el fisioterapeuta 


\section{REFERENCIAS}

Binda, N. U., y Benavent, F. B. (2013). Buscando las ventajas de las diferentes metodologías, (2), 179-188.

Casas, A. S.,y Ramirez, P. C. (2015). Professional skills required in the training of physical therapists in Colombia, 48-52.

Chamorro, Angela Patricia; Paz, Clara Ines; Vernaza, P. (2009). Características laborales de los egresados del programa Cauca, 11, 9-16.

Colombia, C. de. Ley 528 de 1999, 1999 Diario Oficial (1999). Colombia.

De la Torre, 0. L. Y. (2012). Universidad de Guayaquil Facultad de Filosofía, Letras y Ciencias de la Educación. Instituto de post-grado y educación continua programa de maestría en gerencia educativa, 266.

Rodríguez Rojas, Y. L. y Sarmiento Castillo, F. C. (2012). Caracterización de la formación en fisioterapia en países europeos. Método Científico, 6 (1), 58-69.

Ministerio de salud y protección social; Asociación Colombiana de Fisioterapia; Colegio Colombiano de Fisioterapeutas. (2015). Perfil Profesional y Competencias del Fisioterapeuta en Colombia, 62.

Muñoz, R. D.I. (2013). Caracterización del perfil profesional, condiciones laborales y de salud de los fisioterapeutas egresados de la Universidad CES Medellín.

Quiroz, E. C., Durán, E. P., y Quiñones, A. G. (2012). Competencias profesionales en Fisioterapia. A model of assessment based on professional competences in Physiotherapy, 14(23), 155-164.

Vicente, S. Agredo, F. Mina, J. (2015). Perfil profesional y ocupacional de los fisioterapeutas en Colombia. Professional and occupational profile of physiotherapists in Colombia. CES Movimiento y Salud, 3(31), 35-43.

Villabona, E. H., Marina, D., y Lemos, C. (2004). Perfil profesional de los fisioterapeutas egresados de la UIS. 


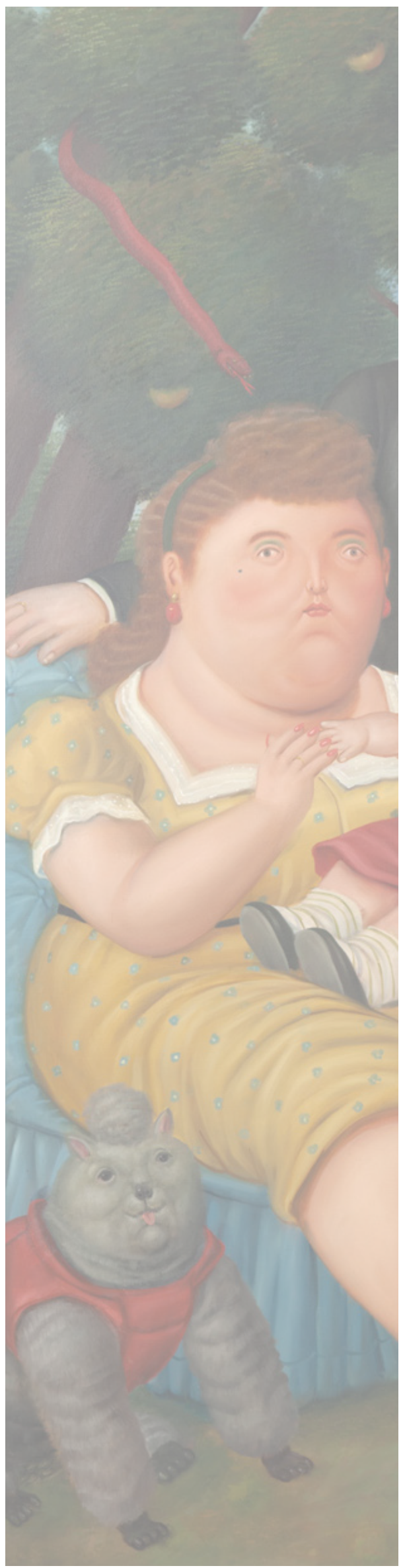

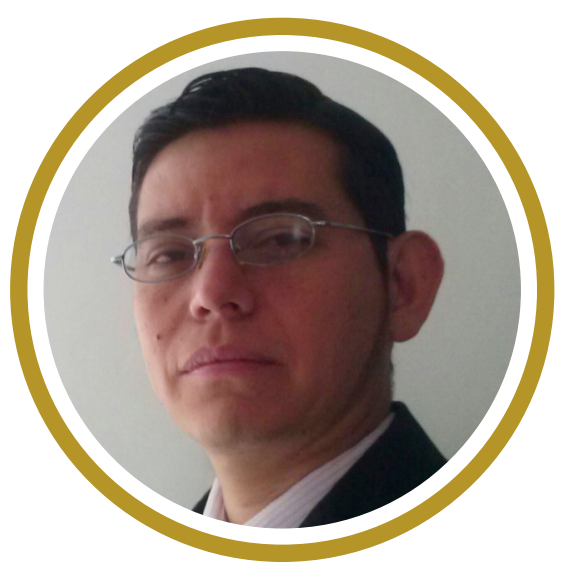

\section{Emerson Julián \\ Rincon Castillo}

Fisioterapeuta egresado de la Universidad Industrial de Santander. Especialista en Rehabilitación Cardiaca y Pulmonar de la Universidad del Rosario. Candidato a Magíster en Educación de la Fundación Universitaria Los Libertadores. Doctor en Educación Atlantic International University. Docente de carrera del Programa de Fisioterapia de la Corporación Universitaria Iberoamericana.

\section{Correo Electrónico:}

rcemersonjulian@gmail.com

Fecha de Recepción: 15/03/20

Fecha de Aprobación: 15/08/20 